\title{
MicroRNA content of horse and human milk exosomes
}

\author{
Sergey Sedykh \\ ICBFM SB RAS, Novosibirsk, Russia \\ sedyh@niboch.nsc.ru
}

\author{
Anna Kuleshova \\ ICBFM SB RAS, Novosibirsk, Russia \\ aekuleshova25@gmail.com
}

Georgy Nevinsky

ICBFM SB RAS, Novosibirsk, Russia nevinsky@niboch.nsc.ru

\begin{abstract}
Exosomes are extracellular vesicles secreted by almost all cell types. Exosomes were isolated from different biological liquids: cell cultures, blood, urine, tears, saliva and milk. Milk is unique source of exosomes. Here we describe microRNA content of horse and human milk exosomes.
\end{abstract}

Keywords - exosomes, milk, microRNA

Motivation and aim

Exosomes are 40-100 $\mathrm{nm}$ diameter natural vesicles, containing CD9, CD63 and CD81 tetraspanins on the surface. It was shown that milk obtained from different sources contain exosomes: human, horse, bovine, rat. Horse milk is a unique source of exosomes since is less allergenic than bovine and not prion prone. Also, horse milk can be obtained in larger amounts than human milk.

\section{Methods}

According to the results, published by the other research groups, milk exosomes contain hundreds and thousands of proteins, mRNA and microRNA molecules. Our recent results show that these numbers may be significantly overestimated.
Isolation of exosomes from sediments obtained after ultracentrifugation with an additional step of gel-filtration allows decreasing the number of proteins, that co-isolate with vesicles. Many microRNAs are described in exosomes, obtained from human and bovine milk.

\section{Results}

Here we show the content of more than two dozens major microRNA molecules, isolated from human and horse milk exosomes on different stages of centrifugations, ultracentrifugations, before and after gel-filtration. According to these data, several microRNAs may be used as indicators of milk exosomes' purity.

\section{ACKNOWLEDGMENT}

The study was funded by the Russian Scientific Foundation (research project 187410055 to S. Sedykh) and by the Program of Fundamental Research of Government Academia (AAAA-A17-117020210023-1 to Prof. G. Nevinsky). 